\title{
A review of the antipatterns detection approaches in object-oriented design
}

\begin{abstract}
Pattern is one of the most simple and powerful techniques to improve the design, and consequently enhance the maintainability, reusability, and reverse engineering. Design pattern detection is a useful technique for gaining knowledge on the design issues of existing systems and improves the system's comprehension, which consequently enhance the software maintainability, reusability, and evolution. Numerous studies have been conducted and many tools have been developed to detect design patterns, whereas only few studies considered the antipattern, which has not been investigated with the same extent of patterns detection. This study presents the antipatterns of object oriented design, their definitions, detecting approaches and issues related to their detection. That provides a clear state of the antipatterns detection and its issues that need to be addressed in the future. The result indicates that the antipatterns detection requires further investigation for several limitations and issues.
\end{abstract}

Keyword: Antipatterns; Object oriented design; Antipatterns detection 